Journal of Applied Analysis

Vol. 10, No. 1 (2004), pp. 105-115

\title{
STABILITY OF THE INTEGRAL CONVOLUTION OF k-UNIFORMLY CONVEX AND k-STARLIKE FUNCTIONS
}

\author{
U. BEDNARZ and S. KANAS
}

Received June 16, 2002 and, in revised form, April 28, 2003

\begin{abstract}
For a constant $k \in[0, \infty)$ a normalized function $f$, analytic in the unit disk, is said to be $k$-uniformly convex if $\operatorname{Re}(1+$ $\left.z f^{\prime \prime}(z) / f^{\prime}(z)\right)>k\left|z f^{\prime \prime}(z) / f^{\prime}(z)\right|$ at any point in the unit disk. The class of $k$-uniformly convex functions is denoted $k-\mathcal{U C V}$ (cf. [4]). The function $g$ is said to be $k$-starlike if $g(z)=z f^{\prime}(z)$ and $f \in k-\mathcal{U C V}$.

For analytic functions $f, g$, where $f(z)=z+a_{2} z^{2}+\cdots$ and $g(z)=$ $z+b_{2} z^{2}+\cdots$, the integral convolution is defined as follows:

$$
(f \otimes g)(z)=z+\sum_{n=2}^{\infty} \frac{a_{n} b_{n}}{n} z^{n} .
$$

In this note a problem of stability of the integral convolution of $k$ uniformly convex and $k$-starlike functions is investigated.
\end{abstract}

2000 Mathematics Subject Classification. 30C45, 30C50, 30C55.

Key words and phrases. $k$-uniformly convex functions, $k$-starlike functions, Hadamard product (or convolution), integral convolution, integral transformation, neighbourhoods of functions, stability of convolution.

ISSN 1425-6908（C) Heldermann Verlag. 


\section{Introduction and definitions}

Let $\mathcal{H}$ denote the class of functions $f$ analytic in the unit disk $\mathcal{U}$

$$
f(z)=z+\sum_{n=2}^{\infty} a_{n} z^{n}
$$

and let $\mathcal{S}$ denote the subclass of functions in $\mathcal{H}$ which are univalent in $\mathcal{U}$. Further, for $k \in[0, \infty)$ let $k-\mathcal{U C V}$ and $k-\mathcal{S} \mathcal{T}$ be the subclasses of $\mathcal{S}$ consisting, respectively, of functions which are $k$-uniformly convex and $k$-starlike in $\mathcal{U}$ defined, respectively, as follows:

$$
\begin{aligned}
& k-\mathcal{U C V}:=\left\{f \in \mathcal{S}: \operatorname{Re}\left(1+\frac{z f^{\prime \prime}(z)}{f^{\prime}(z)}\right)>k\left|\frac{z f^{\prime \prime}(z)}{f^{\prime}(z)}\right|, \quad z \in \mathcal{U}\right\}, \\
& k-\mathcal{S} \mathcal{T}:=\left\{f \in \mathcal{S}: \operatorname{Re}\left(\frac{z f^{\prime}(z)}{f(z)}\right)>k\left|\frac{z f^{\prime}(z)}{f(z)}-1\right|, \quad z \in \mathcal{U}\right\} .
\end{aligned}
$$

Observe that the classes $k-\mathcal{U C V}$ and $k-\mathcal{S T}$ are related by the classical Alexander theorem, that is known also as an equivalence between usual class of convex functions $\mathcal{C V}$ and the class of starlike functions $\mathcal{S T}$. Also note, that the class $k-\mathcal{U C V}$ was defined pure geometrically as a subclass of univalent functions that map each circular arc contained in the unit disc $\mathcal{U}$ with a center $\zeta,|\zeta| \leq k(0 \leq k<\infty)$, onto a convex arc. Classes $k-\mathcal{U C V}$ and $k-\mathcal{S T}$ were introduced by Kanas and Wiśniowska ([4], [6]) and studied in a series of papers $([4],[5],[6],[7])$. Some of properties of $k-\mathcal{U C V}$ and $k-\mathcal{S T}$, in particular, concerning the stability of the Hadamard product, were studied by Bednarz and Kanas in [1].

The convolution, or Hadamard product, of two functions $f$ and $g$ of power series $f(z)=z+a_{2} z^{2}+\cdots$, and $g(z)=z+b_{2} z^{2}+\cdots$, convergent in $\mathcal{U}$, is the function $h=f * g$ with the power series

$$
h(z)=(f * g)(z)=z+\sum_{n=2}^{\infty} a_{n} b_{n} z^{n}, \quad z \in \mathcal{U} .
$$

Convolution has the algebraic properties of ordinary multiplication, the geometric series $K(z)=z+z^{2}+\cdots=\frac{z}{1-z}$ acts as the identity element under convolution: $(f * K)(z)=f(z)$, for any $f \in \mathcal{H}, z \in \mathcal{U}$.

The integral convolution $H=f \otimes g$ is defined by

$$
H(z)=(f \otimes g)(z)=z+\sum_{n=2}^{\infty} \frac{a_{n} b_{n}}{n} z^{n}=\int_{0}^{z} \frac{h(\zeta)}{\zeta} d \zeta, \quad z \in \mathcal{U} .
$$

Note that, if $I$ denotes $I(z) \equiv z$ then

$$
f * I=I \quad \text { and } \quad f \otimes I=I .
$$


Various properties of Hadamard product and integral convolution were studied by several authors, e.g. Robertson ([14]), Pólya and Schoenberg ([9]), Ruscheweyh and Sheil-Small ([13]), Hayman ([3]), Bshouty ([2]). The most famous is Pólya and Schoenberg conjecture ([9]), that the class of starlike functions is preserved by the integral convolution. This conjecture was proved by Ruscheweyh and Sheil-Small ([13]). However as was shown by Hayman ([3]) and Bshouty ([2]) independently, the univalence is not preserved by integral convolution.

In accordance to Ruscheweyh ([11]), let $\mathcal{V}^{*}$ denote the dual set of $\mathcal{V} \subset \mathcal{H}$. Then

$$
\mathcal{V}^{*}=\left\{g \in \mathcal{H}: \frac{(f * g)(z)}{z} \neq 0, \forall f \in \mathcal{V}, \forall z \in \mathcal{U}\right\},
$$

and $\mathcal{V}^{* *}=\left(\mathcal{V}^{*}\right)^{*}$ denote the second dual, which is the smallest of all dual classes containing $\mathcal{V}$. The duality principle states that for compact and complete $\mathcal{V}$ the closed convex hull of $\mathcal{V}$ is the same as the closed convex hull of $\mathcal{V}^{* *}$, so that under fairly weak conditions on $\mathcal{V}$, many extremal problems in $\mathcal{V}$ are solved in $\mathcal{V}^{* *}$ and can be obtained by unified approach.

Dual sets for the classes $k-\mathcal{S T}$ and $k-\mathcal{U C V}$ were found by Kanas, Wiśniowska (cf. [4], [7]). Let us denote the dual set for $k-\mathcal{S} \mathcal{T}$ by $\mathcal{B}$ and for $k-\mathcal{U C} \mathcal{V}$ by $\mathcal{G}$.

Then

$$
f \in k-\mathcal{S} \mathcal{T} \Longleftrightarrow \frac{(f * h)(z)}{z} \neq 0, \forall h \in \mathcal{B}, \forall z \in \mathcal{U}
$$

and

$$
f \in k-\mathcal{U C V} \Longleftrightarrow \frac{(f * h)(z)}{z} \neq 0, \forall h \in \mathcal{G}, \forall z \in \mathcal{U},
$$

respectively (cf. [7]). For $h(z)=z+c_{2} z^{2}+\ldots, h \in \mathcal{B}$ we have the following estimates (cf. [7]),

$$
\left|c_{n}\right| \leq n+(n-1) k, \quad n \geq 2,
$$

and for $h \in \mathcal{G}$

$$
\left|c_{n}\right| \leq n[n+(n-1) k], \quad n \geq 2 .
$$

Further, sufficient conditions to be in $k-\mathcal{S T}$ and $k-\mathcal{U C V}$ are (cf. [7])

$$
\begin{gathered}
\sum_{n=2}^{\infty}[n+(n-1) k]\left|a_{n}\right| \leq 1 \quad \Longrightarrow f \in k-\mathcal{S} \mathcal{T}, \\
\sum_{n=2}^{\infty} n[n+(n-1) k]\left|a_{n}\right| \leq 1 \quad \Longrightarrow \quad f \in k-\mathcal{U C V},
\end{gathered}
$$

respectively. 
For $\delta \geq 0$ Ruscheweyh ([12]) defined $N_{\delta}$ neighbourhood of a function $f(z)=z+\sum_{n=2}^{\infty} a_{n} z^{n}$ by

$$
N_{\delta}(f)=\left\{g(z)=z+\sum_{n=2}^{\infty} b_{n} z^{n} \in \mathcal{H}: \sum_{n=2}^{\infty} n\left|a_{n}-b_{n}\right| \leq \delta\right\} .
$$

By $N_{\delta}(\mathcal{A}), \mathcal{A} \subset \mathcal{H}$, we denote the union of all neighbourhoods $N_{\delta}(f)$ with $f$ ranging over the class $\mathcal{A}$. The quantity $\sum_{n=2}^{\infty} n\left|a_{n}-b_{n}\right|$ can be regarded as the distance between two functions $f$ and $g$ in a some subclass of $\mathcal{H}$, equipped with the pre-norm of functions $F(z)=z+\sum_{n=2}^{\infty} A_{n} z^{n}$ defined as $\|F\|=\sum_{n=2}^{\infty} n\left|A_{n}\right|$. Ruscheweyh proved certain inclusions for the mentioned above neighbourhoods, in particular that $N_{1 / 4}(f) \subset \mathcal{S T}$ holds for all $f \in \mathcal{C} \mathcal{V}$. Problem of neighbourhoods in various classes of functions was also studied in papers [1], [7], [10], [15], [16].

Assume that $A, B$ are subclasses of the class $\mathcal{H}$. Then the set of all function $f * g$ and $f \otimes g$, where $f \in A$ and $g \in B$, will be denoted by $A * B$ and $A \otimes B$, respectively (cf. eg. [13], [15]). Let $A * B \subset C$, the convolution (1.4) is called $C$-stable on the pair of classes $(A, B)$ if there exists $\delta>0$ such that $N_{\delta}(A) * N_{\delta}(B) \subset C$ and $C$-unstable otherwise (cf. [8]). Stability of inclusions for integral convolution is defined in a similar way. The constant $\delta$ which characterizes the stability of Hadamard or integral convolutions is defined as

$$
\delta(A * B, C)=\sup \left\{\delta: N_{\delta}(A) * N_{\delta}(B) \subset C\right\}
$$

and

$$
\delta(A \otimes B, C)=\sup \left\{\delta: N_{\delta}(A) \otimes N_{\delta}(B) \subset C\right\},
$$

respectively. If the related value (1.14) or (1.15) is positive then there exists a neighborhood of the class $A$ and $B$ mapped by the convolution or the integral convolution into $C$.

The stability of the Hadamard or integral convolution can be regarded as a problem of preserving a product of the topology of neighbourhoods in $A$ and $B$ onto the product $A * B$, and $A \otimes B$, respectively.

Problem of the stability of the inclusion for the Hadamard product as well as the integral convolution in the classes of univalent, starlike and convex functions was considered by Nezhmetdinov ([8]). Among other he proved that $\delta(\{I\} *\{I\}, \mathcal{C V})=1$ and $\delta(\{I\} *\{I\}, \mathcal{S})=\sqrt{2}$. Numerous results concerning the stability of the Hadamard product were obtained by Kanas and Bednarz ([1], [7]). In particular in [7] Kanas proved that

$$
N_{1 /[4(k+1)]}(f) \subset k-\mathcal{S} \mathcal{T} \quad \text { for } \quad f \in k-\mathcal{U C V},
$$

that is a k-uniform version of the Ruscheweyh result. Also, we should mention some inequalities about stability of the Hadamard product for functions 
from the classes $k-\mathcal{U C V}$ and $k-\mathcal{S T}$, below:

$$
\begin{aligned}
\delta(\{I\} *\{I\}, k-\mathcal{U C V}) & \geq \frac{1}{\sqrt{k+1}} \\
\delta(\{I\} *\{I\}, k-\mathcal{S T}) & \geq \sqrt{\frac{2}{k+1}} \\
\delta(k-\mathcal{U C V} * \mathcal{C} \mathcal{V}, k-\mathcal{S T}) & \geq \sqrt{4+\frac{1}{2(k+1)^{2}}}-2 .
\end{aligned}
$$

Let $Q[f]$ denote the integral transformation:

$$
Q[f](z)=\int_{0}^{z} \frac{f(t)}{t} d t, \quad z \in \mathcal{U},
$$

where $f \in \mathcal{A} \subset \mathcal{H}$. Also, denote by $Q[A]$ the image of a subclass $A$ under the mapping $Q$. It is well known that $Q[\mathcal{S T}]=\mathcal{S}$, however $Q[\mathcal{S}] \not \subset \mathcal{S}$ (cf. [13], $[2],[3])$. If the inclusion $Q[A] \subset B$ holds, then we can define the constant

$$
\delta(Q[A], B)=\sup \left\{\delta: Q\left[N_{\delta}(A)\right] \subset B\right\}
$$

representing a quantitative characteristic of the stability of the inclusion.

In the present article we consider a problem of a stability of the integral convolution over the class $k-\mathcal{U C V}$ and $k-\mathcal{S T}$. We also study a stability of geometric properties of a function $f$ under the integral transformation $Q[f]$ when $f$ is in $k-\mathcal{U C V}$ or $k-\mathcal{S T}$.

\section{Stability of the integral convolution}

In this section we obtain some results concerning the stability of the integral convolution in the class $k-\mathcal{U C V}$ and $k-\mathcal{S T}$.

In the sequel the following notation will be used: $f(z)=z+\sum_{n=2}^{\infty} a_{n} z^{n}$, $f_{0}(z)=z+\sum_{n=2}^{\infty} a_{0 n} z^{n}, g(z)=z+\sum_{n=2}^{\infty} b_{n} z^{n}, g_{0}(z)=z+\sum_{n=2}^{\infty} b_{0 n} z^{n}$. First of all notice, that by (1.4), (1.5) and (1.20)

$$
(f \otimes g)(z)=\int_{0}^{z} \frac{(f * g)(t)}{t} d t=Q[f * g](z),
$$

and it is easily verified that $Q[k-\mathcal{S T}]=k-\mathcal{U C V}$, therefore

$$
(f \otimes g)(z) \in k-\mathcal{U C V} \Longleftrightarrow(f * g)(z) \in k-\mathcal{S T} .
$$

By the fact $\delta(k-\mathcal{S} \mathcal{T} * \mathcal{C V}, k-\mathcal{S T})=0$, (cf. [7]) and by (2.2), it can be immediately seen that $\delta(k-\mathcal{S} \mathcal{T} \otimes \mathcal{C} \mathcal{V}, k-\mathcal{U C V})=0$. Below, we present other 
stability results over $k-\mathcal{U C V}$ and $k-\mathcal{S T}$, however the authors do not know if they are sharp.

Theorem 2.1. For the integral convolution (1.5) the following inequalities concerning stability in the class $k-\mathcal{U C V}$ and $k-\mathcal{S T}$ are satisfied:

$$
\begin{aligned}
\delta(\{I\} \otimes\{I\}, k-\mathcal{S} \mathcal{T}) & \geq \frac{2}{\sqrt{k+1}}, \\
\delta(\{I\} \otimes\{I\}, k-\mathcal{U C V}) & \geq \sqrt{\frac{2}{k+1}}, \\
\delta(k-\mathcal{U C V} \otimes\{I\}, k-\mathcal{S} \mathcal{T}) & \geq \sqrt{1+\frac{4}{k+1}}-1, \\
\delta(k-\mathcal{S} \mathcal{T} \otimes\{I\}, k-\mathcal{S} \mathcal{T}) & \geq \sqrt{4+\frac{4}{k+1}}-2, \\
\delta(k-\mathcal{U C V} \otimes\{I\}, k-\mathcal{U C V}) & \geq \sqrt{1+\frac{2}{k+1}}-1, \\
\delta(k-\mathcal{U C V} \otimes \mathcal{C V}, k-\mathcal{U C V}) & \geq \sqrt{4+\frac{1}{2(k+1)^{2}}}-2, \\
\delta(k-\mathcal{S} \mathcal{T} \otimes \mathcal{C V}, k-\mathcal{S} \mathcal{T}) & \geq \sqrt{9+\frac{1}{(k+1)^{2}}}-3 .
\end{aligned}
$$

Proof. Making use of (2.2) and inequalities (1.18), (1.19) the relations (2.4), (2.8) follow immediately.

Further, for any $f, g, f_{0}, g_{0} \in \mathcal{H}$ and $h \in \mathcal{B}$ we have

$$
\begin{aligned}
\left|\frac{(f \otimes g * h)(z)}{z}\right| \geq\left|\frac{\left(f_{0} \otimes g_{0} * h\right)(z)}{z}\right|-\left|\frac{\left(f_{0} \otimes\left(g-g_{0}\right) * h\right)(z)}{z}\right| & \\
- & \left|\frac{\left(\left(f-f_{0}\right) \otimes g_{0} * h\right)(z)}{z}\right|-\left|\frac{\left(\left(f-f_{0}\right) \otimes\left(g-g_{0}\right) * h\right)(z)}{z}\right| .
\end{aligned}
$$

Case (2.3). Assume $f_{0}=g_{0}=I$ and let $f, g \in N_{\delta}(I)$, with $\delta$ satisfying (2.3). We shall show that $(f \otimes g * h)(z) / z \neq 0$ for $h \in \mathcal{B}$, or equivalently $f \otimes g \in k-\mathcal{S} \mathcal{T}$ (in view of (1.7)). Observe that, by properties of Hadamard product and integral convolution, we have $\left(f_{0} \otimes\left(g-g_{0}\right) * h\right)(z)=0,((f-$ $\left.\left.f_{0}\right) \otimes g_{0} * h\right)(z)=0$ and $\left(f_{0} \otimes g_{0} * h\right)(z)=I(z)$. Moreover, by the fact that $f, g \in N_{\delta}(I)$ we obtain $\sum_{n=2}^{\infty} n\left|a_{n}\right| \leq \delta$ and $n\left|b_{n}\right| \leq \delta$, therefore $\left|b_{n}\right| / n \leq$ $\delta / n^{2} \leq \delta / 4$ for $n \geq 2$. Hence, in view of (1.9), the inequality (2.10) becomes:

$$
\left|\frac{(f \otimes g * h)(z)}{z}\right|>1-\sum_{n=2}^{\infty} \frac{\left|a_{n}\right|\left|b_{n}\right|\left|c_{n}\right|}{n} \geq 1-\frac{\delta}{4} \sum_{n=2}^{\infty}[n+(n-1) k]\left|a_{n}\right|
$$




$$
\geq 1-(k+1) \frac{\delta}{4} \sum_{n=2}^{\infty} n\left|a_{n}\right| \geq 1-\frac{\delta^{2}}{4}(k+1),
$$

that is nonnegative. It means $|(f \otimes g * h)(z) / z|>0$, so that $f \otimes g \in k-\mathcal{S} \mathcal{T}$, which completes the proof.

Case (2.5). Assume that $f_{0} \in k-\mathcal{U C V}$ and $g_{0}=I$. Also, let $f \in N_{\delta}\left(f_{0}\right)$, $g \in N_{\delta}\left(g_{0}\right)$, with $\delta$ satisfying (2.5). Then, applying (2.10), we obtain

$$
\left|\frac{(f \otimes g * h)(z)}{z}\right|>1-\sum_{n=2}^{\infty} \frac{\left|a_{0 n}\right|\left|b_{n}\right|\left|c_{n}\right|}{n}-\sum_{n=2}^{\infty} \frac{\left|a_{n}-a_{0 n}\right|\left|b_{n}\right|\left|c_{n}\right|}{n} .
$$

Since $f_{0} \in k-\mathcal{U C V} \subset \mathcal{C V}$ then $\left|a_{0 n}\right| \leq 1$. By the fact that $g \in N_{\delta}\left(g_{0}\right)$ we have $\sum_{n=2}^{\infty} n\left|b_{n}\right| \leq \delta$ whence $\sum_{n=2}^{\infty}\left|b_{n}\right|=\sum_{n=2}^{\infty} n\left|b_{n}\right| / n \leq \delta / 2$, for $n \geq 2$. Similarly, since $f \in N_{\delta}\left(f_{0}\right)$ we have $\sum_{n=2}^{\infty}\left|a_{n}-a_{0 n}\right| \leq \delta / 2$. Now, applying above inequalities to (2.11), we obtain for all $z \in \mathcal{U}$

$$
\left|\frac{(f \otimes g * h)(z)}{z}\right| \geq 1-(k+1) \frac{\delta}{2}-(k+1) \frac{\delta^{2}}{4}
$$

that is nonnegative when assuming (2.5), so that $f \otimes g \in k-\mathcal{S T}$.

Case (2.6). Assume $f_{0} \in k-\mathcal{S T}$ and $g_{0}=I$. Similarly as in the previous cases, and in view of (1.7), it suffices to show that for $\delta$ satisfying (2.6) and $h \in \mathcal{B}$ the inequality $|(f \otimes g * h)(z) / z|>0$ holds. Since $f_{0} \in k-\mathcal{S} \mathcal{T} \subset \mathcal{S T}$, then $\left|a_{0 n}\right| \leq n$ and, by virtue of (1.9), the inequality (2.10) takes the form

$$
\begin{aligned}
\left|\frac{(f \otimes g * h)(z)}{z}\right| & >1-\sum_{n=2}^{\infty} \frac{\left|a_{0 n}\right|\left|b_{n}\right|\left|c_{n}\right|}{n}-\sum_{n=2}^{\infty} \frac{\left|a_{n}-a_{0 n}\right|\left|b_{n}\right|\left|c_{n}\right|}{n} \\
& \geq 1-(k+1) \sum_{n=2}^{\infty} n\left|b_{n}\right|-(k+1) \sum_{n=2}^{\infty}\left|a_{n}-a_{0 n}\right|\left|b_{n}\right| \\
& \geq 1-(k+1) \delta-(k+1) \frac{\delta^{2}}{4},
\end{aligned}
$$

which is nonnegative since $\delta$ satisfies (2.6).

Case (2.7). In view of (2.2) and (1.7) it suffices to show that $|(f * g * h)(z) / z|>0$ for $f_{0} \in k-\mathcal{U C V}, f \in N_{\delta}\left(f_{0}\right), g_{0}=I, g \in N_{\delta}\left(g_{0}\right)$ and $h \in \mathcal{B}$. We will proceed as in (2.10). Then

$$
\begin{aligned}
\left|\frac{(f * g * h)(z)}{z}\right| \geq\left|\frac{\left(f_{0} * g_{0} * h\right)(z)}{z}\right|-\left|\frac{\left(f_{0} *\left(g-g_{0}\right) * h\right)(z)}{z}\right| \\
-\left|\frac{\left(\left(f-f_{0}\right) * g_{0} * h\right)(z)}{z}\right|-\left|\frac{\left(\left(f-f_{0}\right) *\left(g-g_{0}\right) * h\right)(z)}{z}\right| .
\end{aligned}
$$


Since $f_{0} \in k-\mathcal{U C V} \subset \mathcal{C V}$, then $\left|a_{0 n}\right| \leq 1$ and

$$
\begin{aligned}
\left|\frac{\left(f_{0} *\left(g-g_{0}\right) * h\right)(z)}{z}\right| & <\sum_{n=2}^{\infty}\left|a_{0 n}\right|\left|b_{n}\right|[n+(n-1) k] \\
& \leq(k+1) \sum_{n=2}^{\infty} n\left|a_{0 n}\right|\left|b_{n}\right| \leq(k+1) \delta .
\end{aligned}
$$

The function $g \in N_{\delta}\left(g_{0}\right)$ so that $n\left|b_{n}\right| \leq \delta$ for all $n \geq 2$, whence $\left|b_{n}\right| \leq \delta / 2$, and therefore

$$
\begin{aligned}
\left|\frac{\left(\left(f-f_{0}\right) *\left(g-g_{0}\right) * h\right)(z)}{z}\right| & <\sum_{n=2}^{\infty}\left|b_{n}\right|\left|a_{n}-a_{0 n}\right|[n+(n-1) k] \\
& \leq(k+1) \frac{\delta^{2}}{2} .
\end{aligned}
$$

Applying (2.13) and (2.14) to (2.12) one obtains

$$
\left|\frac{(f * g * h)(z)}{z}\right| \geq 1-(k+1) \delta-(k+1) \frac{\delta^{2}}{2}
$$

which is nonnegative if $\delta$ satisfies (2.6), so that $f * g \in k-\mathcal{S} \mathcal{T}$, and therefore $f \otimes g \in k-\mathcal{U C V}$.

Case (2.9). Let $f_{0} \in k-\mathcal{S} \mathcal{T}, g_{0} \in \mathcal{C} \mathcal{V}$ and $f \in N_{\delta}\left(f_{0}\right), g \in N_{\delta}\left(g_{0}\right)$ and $h \in \mathcal{B}$. Since $f_{0} \in k-\mathcal{S} \mathcal{T}, g_{0} \in \mathcal{C} \mathcal{V}$ we have $f_{0} * g_{0} \in k-\mathcal{S} \mathcal{T}$ (cf. [5]) or, by (2.2), $f_{0} \otimes g_{0} \in k-\mathcal{U C V}$. Thus, in view of $(1.16)\left|\left(f_{0} \otimes g_{0} * h\right)(z) / z\right|>1 /(4(k+1))$. By the identity $f \otimes g * h=f * g \otimes h$, and the above, the relations (2.10) becomes

$$
\begin{aligned}
\left|\frac{(f \otimes g * h)(z)}{z}\right|> & \frac{1}{4(k+1)}-\sum_{n=2}^{\infty} \frac{\left|a_{0 n}\right|\left|b_{n}-b_{0 n}\right|\left|c_{n}\right|}{n} \\
& -\sum_{n=2}^{\infty} \frac{\left|b_{0 n}\right|\left|a_{n}-a_{0 n}\right|\left|c_{n}\right|}{n}-\sum_{n=2}^{\infty} \frac{\left|a_{n}-a_{0 n}\right|\left|b_{n}-b_{0 n}\right|\left|c_{n}\right|}{n} .
\end{aligned}
$$

The coefficients of $f_{0} \in k$-ST $\subset \mathcal{S T}$ satisfy inequality $\left|a_{0 n}\right| \leq n$ for $n \geq 2$ then, by (1.9) we have:

$$
\begin{aligned}
\sum_{n=2}^{\infty} \frac{\left|a_{0 n}\right|\left|b_{n}-b_{0 n}\right|\left|c_{n}\right|}{n} & \leq \sum_{n=2}^{\infty}\left|b_{n}-b_{0 n}\right|[n+(n-1) k] \\
& \leq(k+1) \sum_{n=2}^{\infty} n\left|b_{n}-b_{0 n}\right| \leq(k+1) \delta .
\end{aligned}
$$


Similarly, since $g_{0} \in \mathcal{C V}$ then $\left|b_{0 n}\right| \leq 1$, whereas $f \in N_{\delta}\left(f_{0}\right)$ gives $\sum_{n=2}^{\infty} n\left|a_{n}-a_{0 n}\right| \leq \delta$, so that $\sum_{n=2}^{\infty}\left|a_{n}-a_{0 n}\right| \leq \delta / 2$. Hence

$$
\begin{aligned}
\sum_{n=2}^{\infty} \frac{\left|b_{0 n}\right|\left|a_{n}-a_{0 n}\right|\left|c_{n}\right|}{n} & \leq \sum_{n=2}^{\infty} \frac{\left|a_{n}-a_{0 n}\right|[n+(n-1) k]}{n} \\
& \leq \frac{1}{2}(k+1) \delta .
\end{aligned}
$$

Finally, we have

$$
\begin{aligned}
\sum_{n=2}^{\infty} \frac{\left|a_{n}-a_{0 n}\right|\left|b_{n}-b_{0 n}\right|\left|c_{n}\right|}{n} & \leq(k+1) \sum_{n=2}^{\infty}\left|a_{n}-a_{0 n}\right|\left|b_{n}-b_{0 n}\right| \\
& \leq(k+1)\left(\delta^{2} / 4\right) .
\end{aligned}
$$

By virtue of (2.16), (2.17), and (2.18) the inequality (2.15) gives

$$
\left|\frac{(f \otimes g * h)(z)}{z}\right| \geq \frac{1}{4(k+1)}-(k+1) \delta-(k+1) \delta / 2-(k+1) \frac{\delta^{2}}{4}
$$

that is nonnegative provided that $\delta$ satisfies the inequality (2.9). From this we conclude $f \otimes g \in k-\mathcal{S} \mathcal{T}$, that completes the proof.

\section{Stability of geometric properties of the integral transformation}

In this section we provide some estimates of radii of $N_{\delta}(f)$ such that the integral operator (1.20) carry the neighborhood into $k-\mathcal{U C V}$ or $k-\mathcal{S T}$, however the authors do not know if the results are sharp.

Theorem 3.1. For the integral representation (1.18) the following inequalities are valid:

$$
\begin{gathered}
\delta(Q[\{I\}], k-\mathcal{S} \mathcal{T}) \geq \frac{2}{k+1} \\
\delta(Q[\{I\}], k-\mathcal{U C V}) \geq \frac{1}{k+1} \\
\delta(Q[k-\mathcal{U C V}], k-\mathcal{U C V}) \geq \frac{1}{4(k+1)} .
\end{gathered}
$$

Proof. By using the Hadamard product (1.4) and the integral convolution (1.5), the transformation (1.20) can be rewritten as:

$$
Q[f](z)=f(z) *\left(z+\sum_{n=2}^{\infty} \frac{z^{n}}{n}\right)=f \otimes\left(z+\sum_{n=2}^{\infty} z^{n}\right)=(f \otimes K)(z) .
$$


Case (3.1). Suppose that $f \in N_{\delta}(I)$ with $\delta$ satisfying (3.1). Then it is easy to see, that for all $h \in \mathcal{B}$ and for all $z \in \mathcal{U}$ we have:

$$
\begin{aligned}
\left|\frac{(Q[f] * h)(z)}{z}\right| & \geq 1-\left|\frac{[(f-I) \otimes K * h](z)}{z}\right| \\
& \geq 1-\sum_{n=2}^{\infty} \frac{\left|a_{n}\right|\left|c_{n}\right|}{n}|z|^{n-1}>1-(k+1) \sum_{n=2}^{\infty}\left|a_{n}\right| \\
& \geq 1-(k+1) \frac{\delta}{2} \geq 0 .
\end{aligned}
$$

The assertion and the above give that $\frac{(Q[f] * h)(z)}{z} \neq 0$ and, consequently, $Q[f] \in k-\mathcal{S} \mathcal{T}$.

Case (3.2). Assuming $h \in \mathcal{G}$ and $f \in N_{\delta}(I)$ with $\delta$ satisfying (3.2) and applying the estimation (1.10), we have

$$
\left|\frac{(Q[f] * h)(z)}{z}\right| \geq 1-\sum_{n=2}^{\infty} \frac{\left|a_{n}\right|\left|c_{n}\right|}{n}|z|^{n-1}>1-(k+1) \sum_{n=2}^{\infty} n\left|a_{n}\right| \geq 1-(k+1) \delta .
$$

The above is nonnegative, so that $Q\left[N_{\delta}(I)\right] \subset k-\mathcal{U C V}$ is valid.

Case (3.3). By the relation $Q[k-\mathcal{S T}]=k-\mathcal{U C V}$ and (1.16) we immediately obtain $Q\left[N_{1 /[4(k+1)]}(k-\mathcal{U C V})\right] \subset k-\mathcal{U C V}$.

Acknowledgment. The authors wish to thank the referee for his valuable suggestions.

\section{References}

[1] Bednarz, U., Kanas, S. Generalized neighbourhoods and stability of convolution for the class of k-uniformly convex and k-starlike functions, Folia Sci. Univ. Tech. Resov. 175, Ser. Math. 23 (1999), 29-38.

[2] Bshouty, D., A note on Hadamard products of univalent functions, Proc. Amer. Math. Soc. 80 (1980), 271-272.

[3] Hayman, W. K., On the coefficients of univalent functions, Proc. Cambridge Philos. Soc. 55 (1959), 373-374.

[4] Kanas, S., Wiśniowska, A., Conic regions and k-uniform convexity, J. Comput. Appl. Math. 105 (1999), 327-336.

[5] Kanas, S., Wiśniowska, A., Conic regions and k-uniform convexity II, Folia Sci. Univ. Tech. Resov. 170 (1998), 65-78.

[6] Kanas, S., Wiśniowska, A., Conic regions and k-starlike functions, Rev. Roumaine Math. Pures Appl. 45(3) (2000), 647-657.

[7] Kanas, S., Stability of convolution and dual sets for the class of $k$-uniformly convex and $k$-starlike functions, Folia Sci. Univ. Tech. Resov. 170 (1998), 51-64. 
[8] Nezhmetdinov, I. R., Stability of geometric properties of convolutions of univalent functions, Russian Math. (Iz. VUZ) 37(11) (1993), 27-34.

[9] Pólya, G., Schoenberg, I. J., Remarks on de la Vallée-Poussin means and convex conformal maps of the circle, Pacific J. Math. 8 (1958), 295-334.

[10] Rahman, Q. I., Stankiewicz, J., On the Hadamard products of schlicht functions, Math. Nachr. 106 (1982), 7-16.

[11] Ruscheweyh, S., Duality for Hadamard product with applications to extremal problems, Trans. Amer. Math. 210 (1975), 63-74.

[12] Ruscheweyh, S., Neighborhoods of univalent functions, Proc. Amer. Math. Soc. 81 (1981), 521-527.

[13] Ruscheweyh, S., Sheil-Small, T., Hadamard product of schlicht functions and the Pólya-Schoenberg conjecture, Comment. Math. Helv. 48 (1973), 119-135.

[14] Robertson, M. S., Applications of a lemma of Fejér to typically real functions, Proc. Amer. Math. Soc. 1 (1950), 555-561.

[15] Stankiewicz, J., Neighbourhoods of meromorphic functions and Hadamard products, Ann. Polon. Math. 46 (1985), 317-331.

[16] Stankiewicz, J., Stankiewicz, Z., Some classes of regular functions defined by convolution, Analytic functions, Błażejewko 1982 (Błażejewko, 1982), Lecture Notes in Math. 1039 (1983), Springer, Berlin, 400-408.

URSZULA BEDNARZ

DEPARTMENT OF MATHEMATICS

TECHNiCAL University of RzEszów

W. POLA 2

PL-35-959 RzEszów, Poland

EMAIL: UBEDNARZ@PRZ.EDU.PL
STANISŁAWA KanAS

DEPARTMENT OF MATHEMATICS

TEChNicAl University of RzeszóW

W. Pola 2

PL-35-959 RzEszów, Poland

EMAIL: SKANAS@PRZ.EDU.PL 